\title{
Abell 43: longest period planetary nebula nucleus variable ${ }^{\star}$
}

\author{
J.-E. Solheim ${ }^{1}$, G. Vauclair ${ }^{2}$, A. S. Mukadam ${ }^{3, \star \star}$, R. Janulis ${ }^{4}$, and V. Dobrovolskas ${ }^{5}$ \\ 1 Institute of Theoretical Astrophysics, University of Oslo, Box 1029 Blindern, 0315 Oslo, Norway \\ e-mail: j.e.solheim@astro.uio.no \\ ${ }^{2}$ Universite Paul Sabatier, Observatoire Midi-Pyrénées, CNRS/UMR5572, 14 Av. Belin, 31400 Toulouse, France \\ e-mail: gerardv@ast.obs-mip.fr \\ 3 Department of Astronomy, University of Washington, Seattle, WA 98195-1580, USA \\ e-mail: anjum@astro.washington.edu \\ 4 Institute of Theoretical Physics and Astronomy of Vilnius University, Gostauto 12, Vilnius 01108, Lithuania \\ e-mail: jr@itpa.lt \\ 5 Observatory of Vilnius University, M.K. Ciurlionio 29, Vilnius, 03100, Lithuania \\ e-mail: vidas.dobrovolskas@ff.vu.lt
}

Received 27 February 2007 /Accepted 11 April 2007

\section{ABSTRACT}

\begin{abstract}
Context. Most PG 1159 stars are hydrogen deficient post-AGB stars on their way to the white dwarf cooling sequence. However, a fraction of them show small amounts of atmospheric hydrogen and are known as the hybrid PG 1159 stars. Both the normal and hybrid PG 1159 stars may reside at the centre of a Planetary Nebula. Some PG 1159 stars exhibit non-radial $g$-mode pulsations, providing us with a means to constrain their fundamental physical parameters, internal structure, and evolutionary status; asteroseismology is the technique of using global stellar pulsations to probe the star's interior.

Aims. Model calculations demonstrate that the PG 1159 instability strip is driven by the partial ionization of carbon and oxygen through the $\kappa$-mechanism. The range of excited frequencies in models is sensitive to the details of the chemical composition in the driving zone. Hydrogen content plays a crucial role in deciding the range of excited frequencies for models of hybrid PG 1159 pulsators. The range of periods observed in all PG 1159 pulsators can then be compared against the range predicted by theoretical models, which are based on observations of their chemical composition and evolutionary status. Our aim is to test the validity of the predicted range of pulsation periods for the hybrid PG 1159 variables, Abell 43 and NGC 7094.

Methods. To achieve this goal, we acquired continuous high speed CCD photometry of the hybrid PG 1159 star Abell 43 using the $2.6 \mathrm{~m}$ Nordic Optical Telescope and the $3.5 \mathrm{~m}$ telescope at Apache Point Observatory. We also observed NGC 7094, a hybrid PG 1159 star with atmospheric parameters similar to Abell 43. We analyzed our data to obtain the pulsation spectra of the two stars.

Results. We detected six significant pulsations in the light curves of Abell 43 with periods between $2380 \mathrm{~s}$ and $6075 \mathrm{~s}$. We were also fortunate to detect low amplitude pulsations in our NGC 7094 data with periods between $2000 \mathrm{~s}$ and $5000 \mathrm{~s}$. The observed range of periods for both stars is consistent with the theoretical predictions.

Conclusions. The range of periods observed in both hybrid PG 1159 stars, Abell 43 and NGC 7094, agree with the theoretical model that the pulsations are driven by the $\kappa$-mechanism induced by the partial ionization of carbon and oxygen.
\end{abstract}

Key words. stars: planetary systems - stars: oscillations - stars: individual: Abell 43 - stars: individual: NGC 7094 central star

\section{Introduction}

Hydrogen deficient hot white dwarf stars constitute the PG 1159 class, and are often found at the centre of planetary nebulae. Abell 43 (= WD 1751+10) is one of the four known hybrid PG 1159 stars, which show evidence of a non-negligible hydrogen abundance in their atmospheres (Napiwotzki \& Schönberner 1991). Miksa et al. (2002) recently published the following atmospheric parameters for Abell 43: $T_{\text {eff }}=110 \mathrm{kK}, \log g=5.7$, $X(\mathrm{H})=0.35, X(\mathrm{He})=0.42$, and $X(\mathrm{C})=0.23$ in mass fractions. Werner \& Herwig (2006) found an even higher carbon abundance from HST ultra-violet observations of Abell 43.

Low carbon abundance determinations by Dreizler et al. (1995) had initially led Quirion et al. (2004) to rule out

^ Based on observations made with the Nordic Optical Telescope, operated on the island of La Palma jointly by Denmark, Finland, Iceland, Norway, and Sweden, in the Spanish Observatorio del Roque de los Muchachos of the Instituto de Astrofisica de Canarias.

$\star \star$ Hubble fellow. pulsations in Abell 43 based on their model calculations. But the recent high carbon abundance measurements led Quirion et al. (2005) to re-evaluate their previous conclusions about Abell 43. They found that the revised models suggested that Abell 43 should exhibit $\ell=1 g$-mode pulsations, driven by the classical $\kappa$-mechanism due to the ionization of carbon K-shell electrons, in the range of $\sim 2604-5529$ s. Córsico et al. (2006) carried out nonadiabatic pulsation calculations for a grid of evolutionary PG 1159 models. They predicted that Abell 43 should exhibit periods in the range of $\sim 2600-7000 \mathrm{~s}$ adopting a $0.530 M_{\odot}$ stellar mass evolutionary track.

Ciardullo \& Bond (1996) were the first to report possible variations in the light curve of Abell 43 with a period of about $2473 \mathrm{~s}$. Schuh et al. (2000) detected a longer period of $5500 \mathrm{~s}$. They also suggested that the $\sim 2500 \mathrm{~s}$ period reported by Ciardullo \& Bond (1996) could simply be an artifact of the $5500 \mathrm{~s}$ period caused by the short duration of the observations. Vauclair et al. (2005) discovered multiple modes in the pulsation spectrum of Abell 43, and reported periods at $2600 \mathrm{~s}$ and $3035 \mathrm{~s}$. 
Table 1. Log of observations of Abell 43.

\begin{tabular}{|c|c|c|c|c|c|}
\hline$\overline{\overline{\text { Date }}}$ & $\begin{array}{l}\text { Start } \\
(\mathrm{UT})\end{array}$ & $\begin{array}{c}\text { Length } \\
\text { (h) }\end{array}$ & $\begin{array}{c}\text { Cycle } \\
\text { time(s) }\end{array}$ & $\begin{array}{r}\text { Data } \\
\text { points }\end{array}$ & Observer \\
\hline $2005-06-29$ & $21: 28$ & 4.40 & 30 & 541 & $\mathrm{JES}^{a}$ \\
\hline 2005-07-01 & $03: 43$ & 1.56 & 20 & 283 & JES \\
\hline $2005-07-01$ & $09: 14$ & 1.54 & 37 & 51 & $\mathrm{ASM}^{b}$ \\
\hline $2005-07-01$ & 21:08 & 7.71 & 20 & 1399 & JES \\
\hline $2005-07-02$ & 21:07 & 7.32 & 20 & 1319 & JES \\
\hline $2005-07-10$ & $07: 24$ & 1.71 & 41 & 110 & ASM \\
\hline
\end{tabular}

${ }^{a}$ J.-E. Solheim. ${ }^{b}$ A. S. Mukadam.

Their observations were based on three individual runs, amounting to a net observation time of $7.5 \mathrm{~h}$ at the $2.6 \mathrm{~m}$ Nordic Optical Telescope (NOT).

In this paper, we report the results from 24 hours of timeseries photometry acquired on Abell 43 in 2005 with the $2.6 \mathrm{~m}$ NOT and the $3.5 \mathrm{~m}$ telescope at Apache Point Observatory (APO). We also report pulsation periods detected in the hybrid PG 1159 star NGC 7094 (=WD 2134+125), which is almost like a spectroscopic twin of Abell 43.

\section{Observations}

We observed Abell 43 for four nights, from the 29th of June to the 2nd of July, 2005 using the $2.6 \mathrm{~m}$ NOT in addition to two short runs with the $3.5 \mathrm{~m}$ telescope at APO on the 1st and 10th of July, 2005. The log of observations is given in Table 1.

We conducted observations at the NOT using the Andalucia Faint Object Spectrograph and Camera (ALFOSC), which is equipped with a thinned $2048 \times 2048$ E2V CCD $42-40$ chip. We observed with the broadband blue W92 filter, which has a $F W H M$ of $275 \mathrm{~nm}$ centered at $550 \mathrm{~nm}$; this enables us to gather a fair amount of flux from the target and minimize the sky contribution at the same time. We acquired suitable flat fields each night just after sunset and determined the dark current for each individual image using the overscan region. We controlled the ALFOSC data acquisition using the software interface tcpcom, defining multiple windows to achieve fast readouts (Østensen 2000). We achieved a net readout time of 4.8 to $5.8 \mathrm{~s}$ for the six separate windows around the target star, three comparison stars, and two blank fields for determining the simultaneous sky brightness. We chose a time resolution of $30 \mathrm{~s}$ during our first night at the NOT telescope, and our exposure time became $23.9 \mathrm{~s}$. We reduced the cycle time to $20 \mathrm{~s}$ on subsequent nights, and achieved exposure times of about 14.1, 14.8, and $14.9 \mathrm{~s}$ for slightly different windowing patterns. With real time processing, we were able to display light curves of the raw and sky-subtracted data during acquisition.

We followed the standard basic reduction procedure, subtracting a dark frame from each image and then flat-fielding it. After these preliminary steps, we computed light curves using aperture photometry for several aperture sizes. We selected the best light curve based on the highest $\mathrm{S} / \mathrm{N}$ ratio. For the observations outlined above, we obtained the best result for an aperture diameter of 24 pixels corresponding to 4.6 arcsec on the sky. We corrected for transparency variations and differential extinction by dividing the target star light curve with the average light curve of the comparison stars. In Fig. 1 we show the light curves from the three longest NOT runs.

The APO observations were acquired with the Seaver Prototype Imaging camera (SPIcam) and a Johnson $B$ filter. We used $3 \times 3$ binning and windowing in order to reduce the read

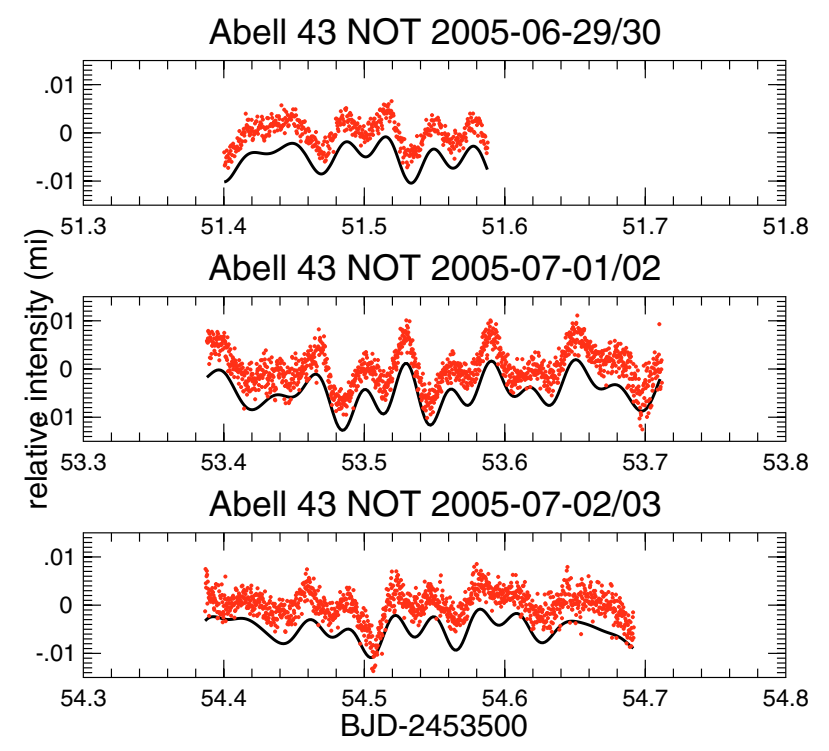

Fig. 1. Light curves of Abell 43 shown for the three long NOT runs. The solid lines are synthetic light curves constructed from the parameters listed in Table 2 , and shifted downward by 0.005 units for clarity.

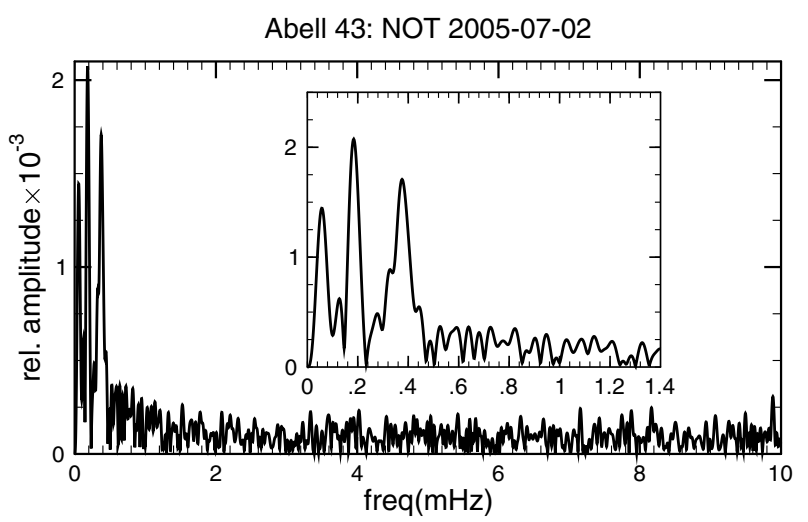

Fig. 2. Pulsation spectrum of the light curve of the NOT run from the 2nd of July, 2005. The insert is a blow up of the low frequency region, that shows the evident lack of excited frequencies above $0.5 \mathrm{mHz}$.

out time to about $25-35 \mathrm{~s}$. The instrument has a read noise of 5 electrons rms, a gain of $1 \mathrm{e} / \mathrm{ADU}$, and a plate scale of 0.42 arcsec/pixel with $3 \times 3$ binning. We used a standard IRAF reduction to extract sky-subtracted light curves from the CCD frames using weighted circular aperture photometry (O'Donoghue et al. 2000).

\section{Hybrid PG 1159 star Abell 43}

\subsection{Single night observations}

We initially computed Discrete Fourier Transforms (DFTs) of the three long light curves acquired using the NOT. We show only the DFT from one of the longest runs in Fig. 2, as the other DFTs look similar. We list the following observed features evident from the DFTs of our NOT data:

- Each pulsation spectrum shows three dominant components, which are relatively stable in frequency, but exhibit variable amplitudes.

- All pulsation spectra have a strong high frequency cut-off above $0.5 \mathrm{mHz}$, beyond which we do not detect any signals. 
Table 2. Identified periodic signals in Abell 43 in the three long NOT runs.

\begin{tabular}{|c|c|c|c|c|c|c|c|c|c|c|c|c|c|c|}
\hline $\begin{array}{l}\text { Date } \\
2005\end{array}$ & $\begin{array}{c}\text { Res. } \\
(\mu \mathrm{Hz})\end{array}$ & $\begin{array}{r}\mathrm{F} 1 \\
(\mu \mathrm{Hz})\end{array}$ & $\begin{array}{l}\text { P1 } \\
\text { (s) }\end{array}$ & $\begin{array}{c}\mathrm{A} 1 \\
\left(\mathrm{mma}^{a}\right)\end{array}$ & $\begin{array}{r}\mathrm{F} 2 \\
(\mu \mathrm{Hz})\end{array}$ & $\begin{array}{l}\text { P2 } \\
\text { (s) }\end{array}$ & $\begin{array}{c}\mathrm{A} 2 \\
(\mathrm{mma})\end{array}$ & $\begin{array}{r}\mathrm{F3} \\
(\mu \mathrm{Hz})\end{array}$ & $\begin{array}{l}\text { P3 } \\
\text { (s) }\end{array}$ & $\begin{array}{c}\mathrm{A} 3 \\
(\mathrm{mma})\end{array}$ & $\begin{array}{r}\mathrm{F} 4 \\
(\mu \mathrm{Hz})\end{array}$ & $\begin{array}{l}\overline{\mathrm{P} 4} \\
\text { (s) }\end{array}$ & $\begin{array}{c}\mathrm{A} 4 \\
(\mathrm{mma})\end{array}$ & 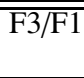 \\
\hline $06-29$ & 63 & 183.3 & 5456 & 2.0 & & & & 363.9 & 2748 & 2.3 & 416.3 & 2402 & 0.8 & 1.98 \\
\hline$\sigma$ & & 1.4 & 41 & 0.1 & & & & 1.2 & 10 & 0.1 & 3.6 & 21 & 0.1 & \\
\hline $07-01$ & 36 & 175.5 & 5698 & 3.1 & 321 & 3118 & 1.0 & 366.4 & 2729 & 2.7 & 408.3 & 2449 & 1.4 & 2.08 \\
\hline$\sigma$ & & 1.2 & 38 & 0.1 & 3.4 & 34 & 0.1 & 0.9 & 10 & 0.1 & 3.4 & 15 & 0.1 & \\
\hline $07-02$ & 38 & 185.2 & 5399 & 2.1 & 325 & 3075 & 0.8 & 368.8 & 2711 & 1.9 & 418.9 & 2387 & 2.0 & 1.99 \\
\hline$\sigma$ & & 1.6 & 47 & 0.1 & 4.4 & 41 & 0.1 & 1.8 & 1.4 & 0.1 & 3.3 & 19 & 0.1 & \\
\hline
\end{tabular}

${ }^{a}$ Relative amplitude $\times 10^{-3}$.

- The light curves are non-stationary ${ }^{1}$ and we have to be cautious in drawing conclusions using standard tools for spectral analysis.

The dramatic cut off implies that we only find pulsations with periods longer than $\sim 2000 \mathrm{~s}$, as predicted both by Quirion et al. (2005) and Córsico et al. (2006). We determined the frequencies, amplitudes, and phases of the significant peaks in the pulsation spectra using a simultaneous non-linear least squares fit (Period04; Lenz \& Breger 2005). To determine which peaks in the DFT constituted real power, we used the method of prewhitening in conjunction with the non-linear least squares analysis. This involves fitting the dominant component and subtracting it from the light curve. If we now compute a DFT of this resultant light curve, the peak corresponding to the dominant mode will be absent. We repeat the procedure for the next highest peak in the pre-whitened DFT, fitting both high-amplitude components simultaneously and then subtracting them from the light curve. The resultant DFT will not show power due to both the highest and second-highest peaks. We continue this process as long as there are well-resolved peaks in the DFT. When the prewhitened DFT does not show any significant power, then we can be sure that we have determined the entire pulsation spectrum using the simultaneous non-linear least squares fit. Figure 3 serves as a demonstration of the prewhitening method.

We show the results of our analysis for the three longest NOT runs in Table 2, that list the significant periods detected with a False Alarm Probability better than 1/100 (Scargle 1982). The uncertainties listed in the table are $1 \sigma$ errors from the non-linear least squares fit. Figure 1 also includes the synthetic light curves constructed from the frequencies, amplitudes and phases determined as above.

It is evident from Table 2 that $\mathrm{F} 1$ varies most in frequency and amplitude from night to night. These variations may be caused by unresolved power. We also notice that the ratio of the frequencies F3:F1 is very close to two on the first and third nights, insinuating a harmonic ratio. The ratio is close to 2.08 on the second night and may perhaps be due to F1 being an unresolved multiplet.

If we compare the periods we detect to those observed earlier, we find that the period $2473 \mathrm{~s}$ discovered by Ciardullo \& Bond (1996) is close to P4, while the period of $5500 \mathrm{~s}$ found by Schuh et al. (2000) is close to P1. The two periods 2600 and $3035 \mathrm{~s}$, discovered by Vauclair et al. (2005) are not too far from P3 and P2. The discrepancies may either be due to insufficient resolution or may perhaps indicate real period changes between the different pulsation modes.

1 When signals detected in a light curve are not present for its entire duration, it is said to be non-stationary in nature.
Abell 43: 2005-06-29 to 07-10

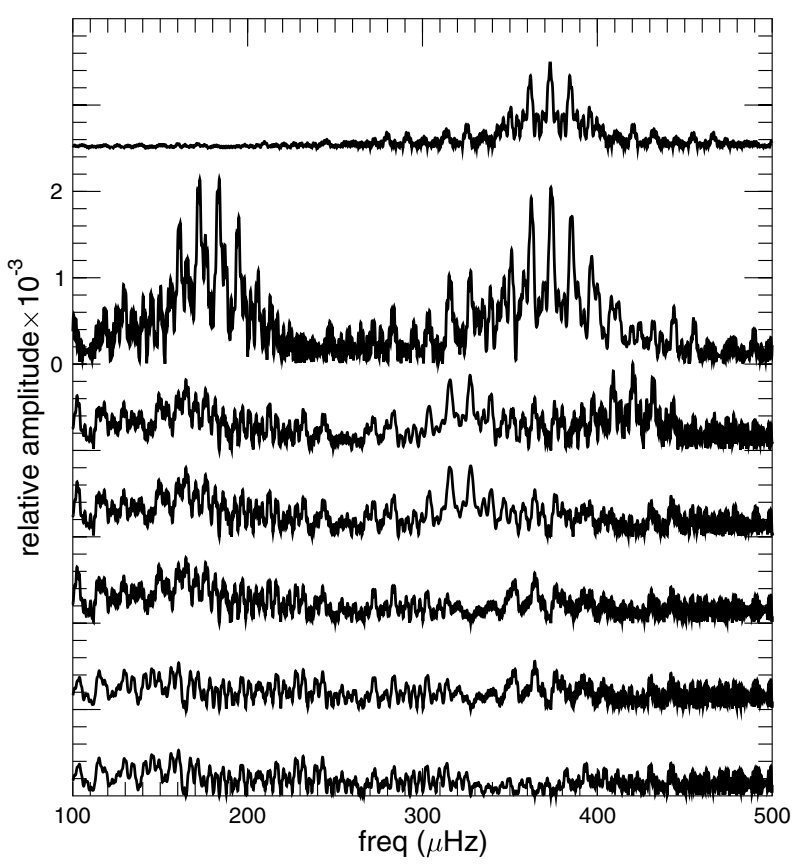

Fig. 3. We show the DFT computed from the combined light curve of five runs used in the analysis. The top curve shows the window function. The second curve shows the DFT of the light curve. The third curve shows the DFT after subtracting out the two highest amplitude peaks. The lower four curves show the DFT after subtracting three, four, five, and six frequencies respectively.

\subsection{Combining all useful observations}

To investigate whether the nightly variations in frequencies and amplitudes were due to unresolved peaks, we re-analyzed the combined light curves with the addition of the APO observations from the 10th of July, 2005. Note that we could not utilize the APO observations from the 1st of July as bad weather rendered them far too noisy for inclusion in our analysis.

We computed a combined DFT again, and used the prewhitening technique in conjunction with the non-linear least squares analysis to arrive at the values listed in Table 3 . We show our final DFT in Fig. 3, along with the different stages of prewhitening. We find that the power seen earlier as F1 split into two individual frequencies $\mathrm{f} 1$ and $\mathrm{f} 2$, while the power at F3 split into $\mathrm{f} 4$ and $\mathrm{f} 5$. Using the new frequency components, we find that $\mathrm{f} 3 / \mathrm{f} 1=1.991$ and $(\mathrm{f} 4+\mathrm{f} 5) / 2 \mathrm{f} 2=2.005$, giving us two ratios of frequencies close to 2 .

Averaging the observations to a sampling time of $200 \mathrm{~s}$, we show the observed light curves along with the synthetic light curves constructed from our fit in Fig. 4; an inspection of this 
Table 3. Identified periodic signals in Abell 43 from the combined data set.

\begin{tabular}{crrcc}
\hline \hline Name & $\begin{array}{r}\text { Freq } \\
(\mu \mathrm{Hz})\end{array}$ & $\begin{array}{r}\text { Period } \\
(\mathrm{s})\end{array}$ & $\begin{array}{c}\text { Amplitude } \\
\left(\mathrm{mma}^{a}\right)\end{array}$ & $\begin{array}{c}\text { Noise } \\
(\mathrm{mma})\end{array}$ \\
\hline $\mathrm{f} 1$ & 164.62 & 6075 & 0.8 & 0.15 \\
$\sigma$ & 0.08 & 3 & 0.1 & \\
$\mathrm{f} 2$ & 183.76 & 5442 & 1.9 & 0.15 \\
$\sigma$ & 0.03 & 1.0 & 0.1 & \\
$\mathrm{f} 3$ & 327.70 & 3051.6 & 0.9 & 0.20 \\
$\sigma$ & 0.07 & 0.7 & 0.1 & \\
$\mathrm{f} 4$ & 363.79 & 2748.8 & 0.9 & 0.20 \\
$\sigma$ & 0.08 & 0.6 & 0.1 & \\
$\mathrm{f} 5$ & 373.12 & 2680.1 & 2.0 & 0.20 \\
$\sigma$ & 0.03 & 0.2 & 0.1 & \\
$\mathrm{f} 6$ & 420.23 & 2379.7 & 1.0 & 0.20 \\
$\sigma$ & 0.06 & 0.4 & 0.1 & \\
\hline
\end{tabular}

${ }^{a}$ Relative amplitude $\times 10^{-3}$.

Abell 43 photometry 2005
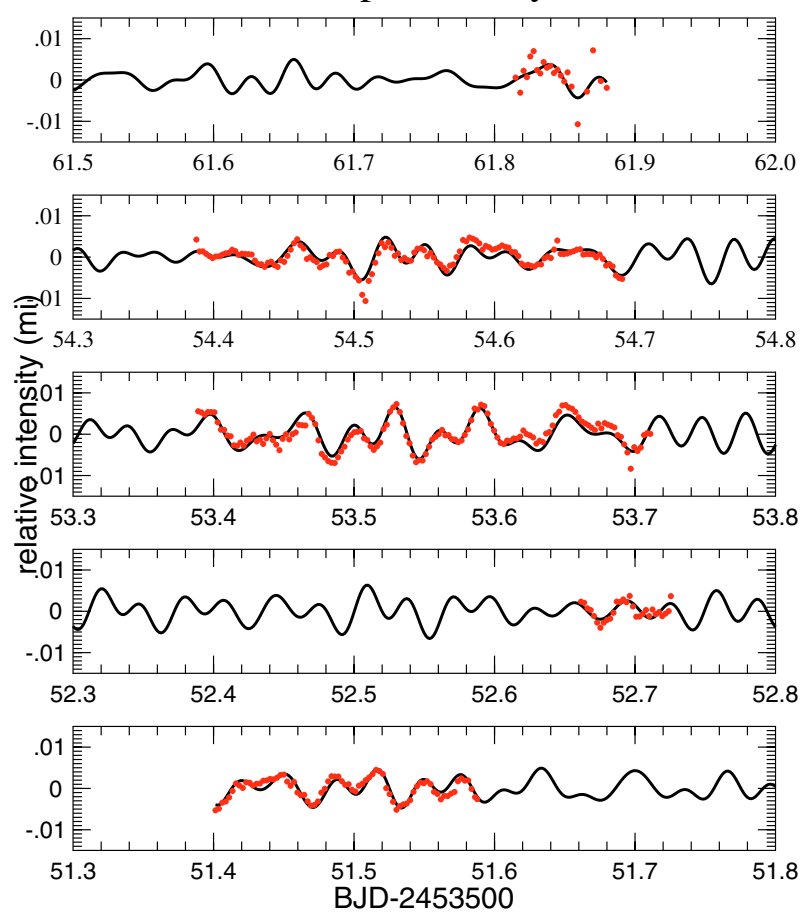

Fig. 4. We show observed light curves, averaged over a sampling time of $200 \mathrm{~s}$, compared with synthetic curves constructed from parameters in Table 3.

figure reveals that the fit is quite good. This tells us that Abell 43 is more stable than the pulsating PNNi with shorter periods, investigated by González Pérez et al. (2006). We also notice how the light curve can change dramatically on short time scales due to beating of the closely spaced modes; this suggests observations over a shorter time span compared to the beat cycle may give wrong results or show no pulsations at all.

The shortest period we detect in our data is $2380 \mathrm{~s}$, which is quite close to the predicted lower limit of $2600 \mathrm{~s}$ for $\ell=1 \mathrm{~g}$-mode pulsations induced by the $\kappa$-mechanism (Quirion et al. 2005; Córsico et al. 2006). The longest period of $6075 \mathrm{~s}$ is slightly longer than predicted by Quirion et al. (2005) but within the range predicted by Córsico et al. (2006). The non stationary nature of the light curves makes it difficult to draw certain conclusions about the frequency ratios close to 2.00. They may
NGC 7094 NOT 2006-11-29

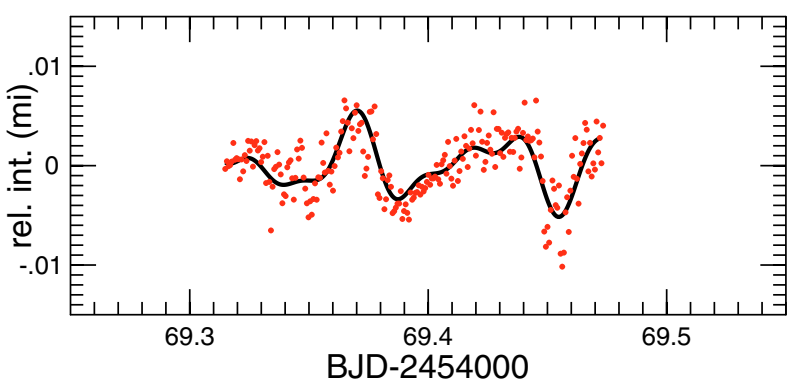

Fig. 5. We show the observed light curve of NGC 7094 with a simulated light curve based on the periods 34,50 , and $83 \mathrm{~min}$.

indicate simple harmonics or non linear resonances as proposed by Buchler et al. (1997).

\section{Hybrid PG 1159 star NGC 7094: Almost a spectroscopic twin of Abell 43}

The hybrid PG 1159 star NGC 7094 shows almost the same spectroscopic parameters as Abell 43, except that 1\% He in Abell 43 has to be replaced by $1 \% \mathrm{O}$ (Miksa et al. 2002). Quirion et al. (2005) propounded that NGC 7094 should pulsate with periods in the range of 2550-5413 s. Previous searches by Ciardullo et al. (1996) and González Pérez et al. (2006) did not reveal any pulsations in the star. The latter observation lasted only $5220 \mathrm{~s}$ and was probably too short to detect pulsations in the predicted range.

In order to scrutinize the variability of NGC 7094, we asked for a service run on the NOT, which can be a maximum of $4 \mathrm{~h}$ long. We obtained a light curve of length $13692 \mathrm{~s}$ with ALFOSC on the 29th of November, 2006. We used the B filter and a window of size $1 \mathrm{k} \times 1 \mathrm{k}$ pixels. We chose an exposure time of $30 \mathrm{~s}$ and the readout time was about the same, giving us a time resolution of $60 \mathrm{~s}$. Figure 5 shows the light curve we obtained from our observations. With the false alarm probability criterion of $1 / 100$ or better, we identified three peaks with periods between $2000 \mathrm{~s}$ and $5000 \mathrm{~s}$. A synthetic light curve constructed from these peaks is also shown in Fig. 5. Longer observing runs are needed to characterize the pulsations better, but this paper serves to establish NGC 7094 as a low-amplitude pulsator.

The range of pulsation periods is shifted toward shorter periods relative to Abell 43. This is predicted by Quirion et al. (2005) and is a consequence of adding a trace of oxygen in their models. The observed lower period limit of $2000 \mathrm{~s}$ is shorter than the theoretical limit of $2550 \mathrm{~s}$ predicted by the same authors.

\section{Conclusions}

For the hybrid PG 1159 star Abell 43, we find six pulsation periods with a range close to that predicted for $\ell=1 \mathrm{~g}$-mode pulsations driven by the $\kappa$-mechanism due to partially ionized carbon (Quirion et al. 2005; Córsico et al. 2006). The light curves are non stationary, but the pulsation frequencies and amplitudes are relatively stable during the 11 day span of the observations. This is different from pulsating PNNi, which showed changes in amplitudes from night to night, and also during the same night (González Peréz et al. 2006).

We have also detected pulsations in the hybrid PG 1159 star NGC 7094 which is an approximate spectroscopic twin of Abell 43. The observed pulsations lie in the range 
of $2000-5000 \mathrm{~s}$, in a band of periods shifted to shorter values than for Abell 43. This agrees with theory and occurs when a small amount of oxygen is added to the model pulsator (Quirion et al. 2005), although the lowest period observed is somewhat shorter than predicted for NGC 7094.

Acknowledgements. The data presented here have mostly been acquired using ALFOSC, which is owned by the Instituto de Astrofisica de Andalucia (IAA) and operated at the Nordic Optical Telescope under agreement between IAA and the NBIfAFG of the Astronomical Observatory of Copenhagen. We would like to specially thank the NOT staff for their service observations of NGC 7094 resulting in the detection of a new pulsator. The rest of the data are based on observations obtained with the Apache Point Observatory 3.5-meter telescope, which is owned and operated by the Astrophysical Research Consortium. Support for a fraction of this work was provided by NASA through the Hubble Fellowship grant HST-HF-01175.01-A awarded by the Space Telescope Science Institute, which is operated by the Association of Universities for Research in Astronomy, Inc., for NASA, under contract NAS 5-26555. We also thank the anonymous referee for suggesting a number of improvements to the first version of this manuscript.

\section{References}

Buchler, J. R., Goupil, M.-J., \& Hansen, C. J. 1997, A\&A, 321, 159 Ciardullo, R., \& Bond, H. E. 1996, AJ, 111, 2332

Córsico, A. H., Althaus, L. G., \& Miller Bertolami, M. M. 2006, A\&A, 458, 259 Dreizler, S., Werner, K., \& Heber, U. 1995, in White Dwarfs, ed. D. Koester, \& K. Werner, Lecture Notes in Physics (Berlin: Springer Velag), 443, 160 González Pérez, J. M., Solheim, J.-E., \& Kamben, R. 2006, A\&A, 454, 527 Lenz, P., \& Breger, M. 2005, Comm. in Asteroseismology, 146, 53

Miksa, S., Deetjen, J. L., Dreizler, S., et al. 2002, A\&A, 389, 953 Napiwotzki, R., \& Schönberner, D. 1991, A\&A, 249, L16

O’Donoghue, D., Kanaan, A., Kleinman, S. J., Krzesinski, J., \& Pritchet, C. 2000, Baltic Astron., 9, 375

Østensen, R. H. 2000, Ph.D. Thesis, University of Troms $\emptyset$, Norway

Quirion, P.-O., Fontaine, G., \& Brassard, P. 2004, ApJ, 610, 436

Quirion, P.-O., Fontaine, G., \& Brassard, P. 2005, A\&A, 441, 231

Scargle, J. D. 1982, ApJ, 263, 835

Schuh, S., Dreizler, S., Deetjen, J. L., Heber, U., \& Geckeler, R. D. 2000, Baltic Astron., 9, 395

Vauclair, G., Solheim, J.-E., \& Østensen, R. H. 2005, A\&A, 433, 1097

Werner, K., \& Herwig, F. 2006, PASP, 118, 183 